\title{
CORRELATION ANALYSIS OF FLAG LEAF WITH YIELD IN SEVERAL RICE CULTIVARS
}

\author{
Md. Asadur Rahman, M. E. Haque, B. Sikdar, Md. Asadul Islam and Muhammad Nurul Matin* \\ Department of Genetic Engineering and Biotechnology, University of Rajshahi, Rajshahi-6205, Bangladesh \\ *Corresponding author. e-mail: nmatin2@yahoo.com
}

\begin{abstract}
The uppermost leaf below the panicle is the flag leaf that provides the most important source of photosynthetic energy during reproduction and grain filling, thereby has great impact in panicle development and grain yield in rice. In the present investigation in order to explore the relationship between grain yield and flag leaf parameters, yield composition, length and width of the flag leaf, and panicle length were measured in some rice cultivars. Statistical analysis indicated that flag leaf length was positively correlated with panicle length for the studied cultivars demonstrating higher grain yield. Chlorophyll measurement indicated that flag leaf contained more chlorophyll than penultimate leaf. Yield of all the cultivars upon excision of flag leaf was also compared. Removal of flag leaf led to a decline in the seed-setting rate which eventually reduced the grain yield. Besides this, variable pollen viability was also noticed in the different cultivars.
\end{abstract}

Keywords: Chlorophyll, flag leaf, panicle, pollen grain, yield.

সারাংশ: ধানের শীভের নিচের সর্বপ্রথম পাতাটিকে ফ্লাগ পাতা বলা হয় যা ধানের দানা পূর্ণতার সময় সালোকসংশ্লেষণের শক্তি উৎপাদনে মূখ্য ভূমিকা পালন করে। ধানের শীভের গঠন ও ধানের ফলনে ফ্লাগ পাতার ভূমিকা অনন্য। এই গবেষণায় ধানের ফলন ও ফ্লাগ পাতার মধ্যকার সমপর্ক দেখার জন্য কিছু ধানের জাতে ফলনের প্রভাবক, ফ্লাগ পাতার দৈর্ঘ্য ও প্রস্থ এবং ধানের শীভের দৈর্ঘ্যের পরিমাপ করা হয়। গবেষণা ফলাফনে দেখা যায়, সকল জাতের ক্ষেত্রেই ফ্লাগ পাতার দৈর্ঘ্য ও শীযের দৈর্ঘ্যের সমপর্ক ধনাত্বক। ফ্লাগ পাতা ও তার নিচের পাতার ক্লোরোফিলের মাত্রা পরিমাপ করে দেখা যায় তুলনামূলকভাবে ফ্লাগ পাতায় ক্লোরোফিলের পরিমাণ বেশি। কিছু ধানের ফ্লাগ পাতা কেটে দিয়ে তার ফলন পরিমাপ করে ফ্লাগ পাতা না কাটা ধানের তুলনায় কম পাওয়া গেছে। এছাড়াও ধানের বিভিন্ন জাতের ক্ষেত্রে পরাগরেণুর সক্ষমতায় ভিন্নতা পরিলক্ষিত হয়।

\section{Introduction}

Rice is one of the most important staple foods for more than two billion peoples of the world (FAO, 1995). Rice consumers are increasing day by day and the number will probably double by the year of 2020 (Khush and Toenniessen, 1991). Rice is the main food and major energy source for the population of Bangladesh which covers $80 \%$ of the total cropped area as about 12 million ha (BRRI 1999). One of the important aims for growing rice is the yield. However, grain yield is a complex trait and genetic control of grain yield is a series of complex biochemical and physiological processes (Ashraf et al. 1994). Photosynthesis of carbohydrate is the primary source of grain yield in rice. Grain filling is sustained by current photosynthesis of the upper parts of the plant, i.e. the flag leaf and penultimate leaves and the ear (Tambussi et al. 2007). Plant leaves being the organ of photosynthesis is considered to be the important determinant and characterized for higher photosynthetic capacities (Asana, 1968).

The uppermost leaf below the panicle is the flag leaf that provides the most important source of photosynthetic energy during reproduction. Flag leaf is metabolically active and has proved that the flag leaf, stem and head are the closest source food to the grain (Asana, 1968; Ramadas and Rajendrudu, 1977). Flag leaf assigned an important role in terms of supply of photosynthates to the grains (Asana, 1968), in grain yield (Sheela et al. 1990; Raj and Tripathi, 2000) and in enhancing productivity (Padmaja Rao, 1991). The grain yield and yield related traits were positively related to flag leaf area (Ashrafuzzaman, et al. 2009). The top three leaves contribute most to grain yield (Yoshida, 1981; Misra, 1987). Intensive study was done on rice yield after cutting flag leaf and nearby leaf (Aboukhalifa et al. 2008). Considering the importance of leaf on grain yield it is prerequisite to analyze the morphological and the physiological characteristics of functional leaves to improve grain yield in rice (Yue et al. 2006).

The photosynthetic pigment chlorophyll is another important molecule associated with photosynthesis in plant leaves that directly affects the biomass (Shen 1980; Ohno 1976; Chen et al. 1995) and grain yield of crops (Xu and Shen, 1994). Chlorophyll is positively correlated with photosynthetic rate (Davis et al. 1979). Ohno (1976) also reported significant differences with photosynthetic rate in rice and suggested high yielding rice could be developed by selecting varieties with high photosynthesis.

Besides the leaf, pollen viability is critically important for pollination and thereby seed setting in the plants. Several researchers have worked on pollen viability analysis through image quality and processing (Ortega et al. 2011).

The aim of this study was to understand the genetic basis of the traits and their possible role in genetic improvement associated with grain yield in rice. The 
rice yield is far below in Bangladesh than the requirement for the population. Meeting this challenge will require efforts in multiple areas, one of which will be the development of high yielding varieties that are better adapted to wide range of environment. Genotype greatly affects grain yield and yield components. Therefore, effective plant selection characters that are associated with yield are obligatory. Correlation analysis and direct and indirect effects of yield components provides the basis for successful breeding plan (Choudhry et al. 1986). Thus, the objective of this study was to determine the correlation analysis of yield and yield contributing characters in rice. Therefore efforts were made to relate the flag leaf area with yield parameters viz., number of panicles, panicle length, number of grains per panicle, grain weight, grain yield per plant, in order to assess and identify the productive cultures for selection. Moreover, chlorophyll content of the leaf and the effect of leaf cutting on the yield components were elucidated as well as pollen viability were analyzed in rice cultivars.

\section{Materials and Methods}

Plant materials and data collection: Mature seeds of rice cultivars BR3, BR4, BR11, BRRI dhan28, BRRI dhan29, BRRI dhan34, and BRRI dhan37 were collected from regional station of Bangladesh Rice Research Institute (BRRI, Rajshahi) and were germinated in pots on May 2011. Thirty day old seedlings were transplanted in the research field of Rajshahi University, Bangladesh using randomized block design with three replications in $2 \mathrm{~m}^{2}$ plot. In every plot 10 rows each containing 10 single seedlings of all the cultivars were transplanted. Spacing between plants and rows was kept 15 and $30 \mathrm{~cm}$, respectively. Standard agronomic practices compatible to this argoecological zone were adapted to ensure good crop growth. At maturity 10 guarded plants were randomly selected from each replication and data were collected for plant height, flag leaf length, days to heading, panicle length, grain per panicle and other yield contributing characters including grain weight, and grain yield per plant at harvest.

Flag leaf excisions: Flag leaf (FL) was excised at the base during heading stage from some of the plants of the studied cultivars and let it to maturity and data were collected to study the effect of FL cutting on grain yield. After harvesting weight of 100 grains was measured using electric balance.
Data analysis: From each replication 10 hills those produced more than 10 tillers were randomly selected. Data on leaf and panicle were taken from10 tillers/hill and average was calculated with standard error $( \pm)$. The mean performance of individual genotype over three replications was pooled and employed for statistical analysis. Correlation coefficient was calculated and the estimated values were compared with tabulated values to test the significance. ANOVA was calculated to test the significance of differences among parameters. Pearsonian coefficient of correlation ( $r$ ) was measured as follows:

$$
r=\frac{\sum x y-\frac{\sum x \sum y}{n}}{\sqrt{\sum x^{2} \cdot \frac{(\Sigma x)^{2}}{n}} \times \sqrt{\sum y^{2} \cdot \frac{(\Sigma y)^{2}}{n}}}
$$

Here, x; flag leaf length, y; panicle length.

To measure the accuracy of significance of $r, t$ test was done using the following formula:

$$
\mathbf{t}=\frac{\mathrm{r} \sqrt{ } \mathrm{n}-2}{\sqrt{ } 1-\mathrm{r}^{2}}
$$

Chlorophyll content measurement: For chlorophyll content measurement leaf samples from FL and penultimate leaves (PL) from the tested cultivars were collected at anthesis period. Total chlorophyll was measured using a spectrophotometer with the following procedure. From fresh and fully expanded leaves $20 \mathrm{mg}$ leaf tissue was prepared and extracted with $1 \mathrm{ml}$ of $95 \%$ ethanol in a tube at $60^{\circ} \mathrm{C}$ for overnight. The extract was measured at wavelengths of both 645 and $663 \mathrm{~nm}$ with T80 UV/VIS Spectrophotometer (PG Instrument Ltd.). Chlorophyll $a$, chlorophyll $b$ and total chlorophyll contents were calculated using MacKinney's (1941) specific absorption coefficients as described by Chory et al. (1989), in which chlorophyll $a=12.72\left(\mathrm{~A}_{663}\right)$ $2.59\left(\mathrm{~A}_{645}\right)$, chlorophyll $b=22.88\left(\mathrm{~A}_{645}\right)-4.68\left(\mathrm{~A}_{6663}\right)$, and total chlorophyll $=20.29\left(\mathrm{~A}_{645}\right)+8.02\left(\mathrm{~A}_{663}\right)$. The total chlorophyll per gram tissue was converted with the formula of, total chlorophyll $(\mathrm{mg} / \mathrm{g})=$ chlorophyll (mg/L) X 0.001 (L) / fresh weight (g). Three biological replicates were used for this experiment.

Pollen viability analysis: Chemicals including 95\% ethanol, Lugols solution containing iodine potassiumiodide $\left(\mathrm{I}_{2} \mathrm{KI}\right)$ and distilled water were used. Three healthy flowering panicles from each plant were selected for pollen viability testing. Five spikelets from each panicle were collected. Open anthers were placed on one drop of demineralized water on a glass slide and 
were macerated with needle to release the pollen grains, followed by staining with a $1 \% \mathrm{I}_{2} \mathrm{KI}$ solution. The $\mathrm{I}_{2} \mathrm{KI}$ solution was prepared by dissolving $1 \mathrm{~g}$ of Iodine and $2 \mathrm{~g}$ of Potassiumiodide in $100 \mathrm{ml}$ of water (Lopez and Virmani, 2000). Pollen viability was studied under microscope (LABOMED, USA) in LP Semi plan Achro $40 \mathrm{X}$ and differenciation was made between viable and nonviable one.

\section{Results}

Flag leaf length positively correlates with yield: Length of flag leaf and panicle of two rice cultivars, BR11 and BRRI dhan28 were measured and correlation between the characters was calculated. The correlation between FL and yield was positive and highly significant. Plants with grater FL length had elongated panicle length, thus producing increased number of primary and secondary rachis resulted in increased number of grain in the panicle that ultimately improved the yield of the cultivar (Table 1). Statistical analysis showed that the value of correlation coefficient $r$ was 0.792749 and 0.971915 for BR11 and BRRI dhan28, respectively. Calculated value was greater than the tabulated value $(0.735$ at $9 \mathrm{df})$, indicating the correlation among parameters for both varieties were significant at $1 \%$ level of significance (Fig. 2). Furthermore, accuracy of significance level was confirmed using t-test. The calculated value of $t$ was 3.678 and 11.681 for BR11 and BRRI dhan28, respectively, which is greater than the tabulated value (3.25 at $9 \mathrm{df}$ ) indicating the result was significant at 1\% level of significance.

Table 1 Data on flag leaf length, penultimate leaf length and panicle length of the variety BR11 and BRRI dhan28.

\begin{tabular}{ccccccc}
\hline & \multicolumn{3}{c}{ BR11 } & \multicolumn{3}{c}{ BRRI dhan28 } \\
\cline { 2 - 7 } Plant no. & FL & PL & Panicle & FL & Length (cm) & PL \\
\cline { 2 - 7 } 01 & $21.3 \pm 1.3$ & $36.1 \pm 1.5$ & $18.3 \pm 1.0$ & $22.1 \pm 2.1$ & $33.5 \pm 2.8$ & $19.7 \pm 2.5$ \\
02 & $25.7 \pm 1.08$ & $34.0 \pm 2.1$ & $18.5 \pm 2.1$ & $18.9 \pm 2.3$ & $32.7 \pm 2.2$ & $17.5 \pm 2.1$ \\
03 & $28.28 \pm 1.3$ & $31.0 \pm 1.5$ & $20.3 \pm 1.8$ & $27.6 \pm 2.9$ & $35.7 \pm 2.7$ & $25.3 \pm 1.9$ \\
04 & $37.28 \pm 2.1$ & $44.7 \pm 2.8$ & $35.0 \pm 2.2$ & $31.9 \pm 2.2$ & $39.8 \pm 1.7$ & $28.8 \pm 2.3$ \\
05 & $18.82 \pm 1.1$ & $30.7 \pm 2.9$ & $17.5 \pm 1.5$ & $21.6 \pm 1.9$ & $30.7 \pm 1.2$ & $16.6 \pm 1.8$ \\
06 & $37.84 \pm 1.9$ & $38.6 \pm 2.2$ & $33.9 \pm 2.5$ & $32.5 \pm 2.2$ & $41.9 \pm 2.4$ & $29.4 \pm 1.7$ \\
07 & $37.59 \pm 2.3$ & $47.0 \pm 3.1$ & $33.4 \pm 1.9$ & $31.8 \pm 1.5$ & $45.2 \pm 2.1$ & $27.5 \pm 2.9$ \\
08 & $25.9 \pm 2.5$ & $36.1 \pm 1.9$ & $19.8 \pm 2.4$ & $29.6 \pm 2.3$ & $40.2 \pm 2.9$ & $25.9 \pm 2.2$ \\
09 & $24.13 \pm 1.2$ & $32.8 \pm 2.7$ & $22.6 \pm 1.6$ & $24.3 \pm 2.1$ & $31.7 \pm 2.0$ & $22.5 \pm 2.6$ \\
10 & $35.5 \pm 1.8$ & $42.9 \pm 2.0$ & $31.6 \pm 2.7$ & $19.1 \pm 2.3$ & $32.4 \pm 2.1$ & $18.4 \pm 2.1$ \\
\hline
\end{tabular}

Randomly 10 plants were selected from each replication where each hill produced more than 10 tillers. Data is an average of 10 tillers/hill. Standard error is demonstrated with \pm value. FL; flag leaf, PL; penultimate leaf.

Table 2 Total chlorophyll content of the flag leaf and penultimate leaf of the studied five cultivars of rice.

\begin{tabular}{lccc}
\hline \multirow{2}{*}{ Genotype } & \multicolumn{3}{c}{ Total chlorophyll content (mg/g) } \\
\cline { 2 - 4 } & Flag leaf & $\begin{array}{c}\text { Penultimate } \\
\text { leaf }\end{array}$ & F value \\
\hline BR 3 & 50.54 & 37.61 & \\
BR 4 & 49.21 & 36.06 & \\
BR 11 & 41.13 & 32.18 & $22.7^{* *}$ \\
BRRI dhan34 & 54.22 & 19.59 & \\
BRRI dhan37 & 42.08 & 33.82 & \\
\hline
\end{tabular}

** Significant at $1 \%$ level
Table 3 100- grain weight of the rice cultivars in normal condition and after removal of flag leaf.

\begin{tabular}{lccc}
\hline \multirow{2}{*}{ Genotype } & \multicolumn{3}{c}{100 -grain weight (g) } \\
\cline { 2 - 4 } & Control \pm sd & Treated \pm sd & t-value \\
\hline BR-3 & $2.75 \pm 0.21$ & $1.4 \pm 0.16$ & $15.47^{* *}$ \\
BR-4 & $2.60 \pm 0.19$ & $1.7 \pm 0.18$ & $10.22^{* *}$ \\
BR-11 & $2.57 \pm 0.23$ & $1.8 \pm 0.1 .0$ & $10.26^{* *}$ \\
BRRI dhan34 & $3.1 \pm 0.14$ & $1.65 \pm 0.17$ & $15.36^{* *}$ \\
BRRI dhan37 & $2.31 \pm 0.21$ & $1.5 \pm 0.16$ & $9.64^{* *}$ \\
\hline
\end{tabular}

Control; plants grown naturally, Treated; plants grown nearby the control plant but flag leaf was removed at the anthesis stage and let it to grow to maturity. After harvesting 100 grains was collected from 5 panicles of one plant and weight was measured in gram. Three replications were used. Data following the marks ** are significantly higher than the treated at 0.01 probability levels based on t-test. 
Leaf chlorophyll contributes to yield: Chlorophyll content of FL and PL from the studied five cultivars was measured. Results indicated that FL contained increased amount of total chlorophyll than that of PL for all of the studied cultivars (Fig. 1 and Table 2). The difference was more than two fold in BRRI dhan34. Among the cultivars, BRRI dhan34 showed the highest $(54.22 \mathrm{mg} / \mathrm{g}) \mathrm{FL}$ chlorophyll content than the others, whereas, BR3 showed maximum PL chlorophyll content (Table 2). ANOVA showed that mean chlorophyll content among the cultivars varied significantly suggesting that the studied genotypes were genetically variable regarding chlorophyll content. Chlorophyll content had the strong and positive correlation with yield. Increased grain yield was observed when chlorophyll was higher as found in the BRRI dhan34, whereas, lower grain yield was observed at lower chlorophyll content as found in the cv.BR11 (Table 2 and 3).

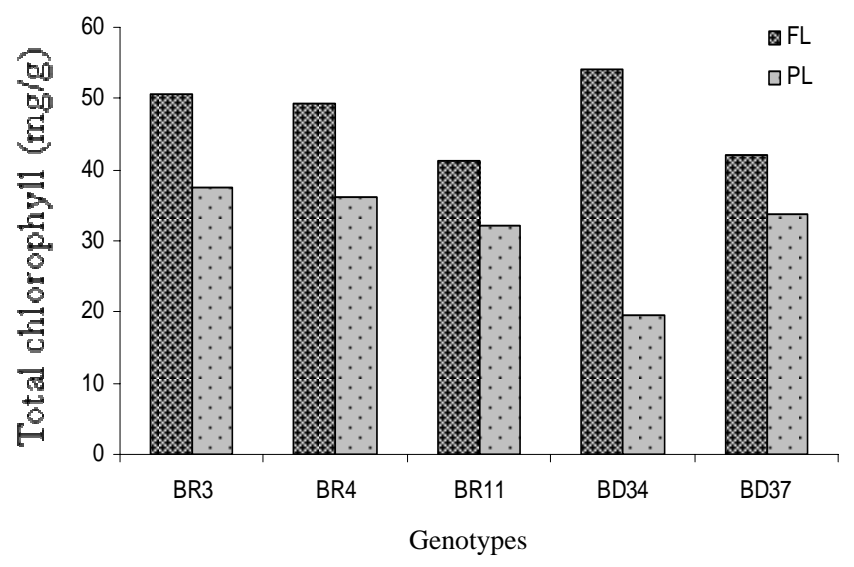

Fig. 1 Frequency distribution of total chlorophyll content in the rice cultivars grown under normal environmental conditions.
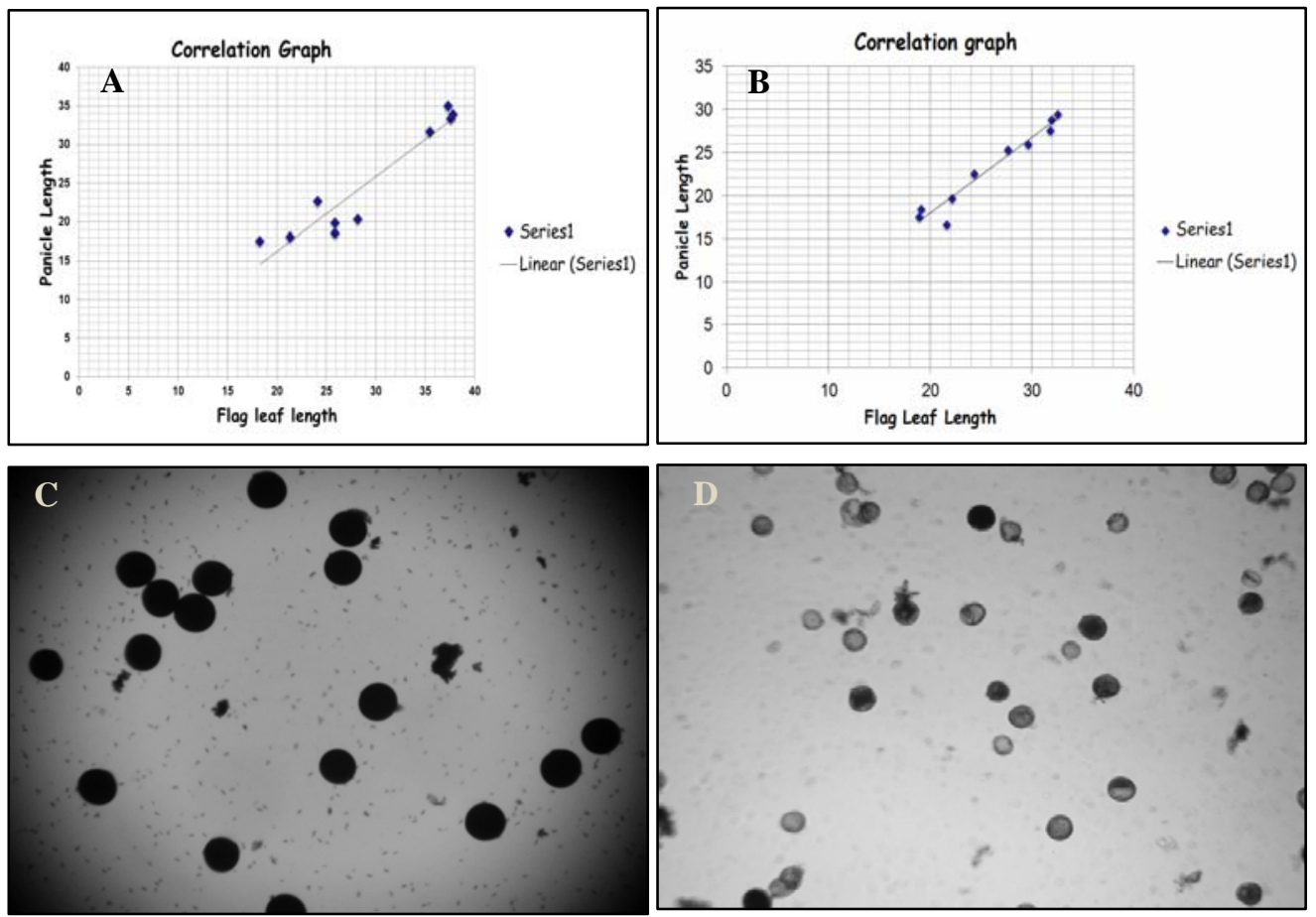

Fig. 2 Correlation graphs and pollen viability study. A. Correlation between flag leaf length and panicle length of the rice cultivar BR 11 . B. Correlation between flag leaf length and panicle length of the rice cultivar BRRI dhan28. C. Viable pollens and D. Non viable pollens observed in 40X objectives. 
Flag leaf cutting affect plant yield: The flag leaves were excised after the emergence of panicle from some of the selected plants of all the examined cultivars and let it to grow. Phenotypic observation indicated various defects existed in the leaf cut plants throughout maturation including late maturation, decaying, shrunken and reduced grain size, as well as increased sterility. Moreover, panicle length and branching were reduced. As a whole, those deformities acted as potential factors for the reduction of yield of the rice cultivars. After harvesting 100 grains weight from leaf cut plant was measured and significant reduction of weight was observed compare to 100 grains weight of the control plant (Table 3). Among cultivars the 100 grain weight of leaf cut plants was reduced about two fold in the BR3 and BRRI dhan34 from those of uncut plants (Table 3).

Pollen viability varies among cultivars: Pollen viability was tested using $\mathrm{I}_{2} \mathrm{KI}$ solution from 10 microscopic focuses. Viable pollen grains were filled with large and ball-like intracellular proteins and non-viable pollen grains were wrinkled and empty and less intracellular proteins were present (Fig. 2). Among them BR3 had the highest percentage of pollen viability (91\%), whereas, BR4, BR11, BRRI dhan34 and BRRI dhan37 showed the pollen viability of $65 \%, 82 \%, 70 \%$ and $80 \%$, respectively.

\section{Discussion}

Present study indicated that flag leaf length was positively correlated with panicle length thereby was correlated with the grain yield which showed the similarity with the results of $\mathrm{Li}$ et al. (1998), where found that large leaf length, leaf width, and leaf area contributed to increased spikelet number per panicle. Bing et al. (2006) found that potential yield and spikelet number were positively correlated with length, width and area of flag leaf. Thereby indicating, it is possible to improve grain yield by genetic improvement of length, width and area of flag leaf. Genetic analysis of the morphological and physiological characteristics of functional leaves, especially flag leaf is found important for rice improvement program (Yue et al. 2006). It is noticed that when FL length is high the panicle length is also high. In case of BR11 when the average FL length was 21.33, 25.90, 28.19, 37.33, 18.28, 37.84, 37.59, 25.90, 24.13, $35.50 \mathrm{~cm}$, then the average panicle length was $18.03,18.54,20.32,34.98,17.52,33.87,33.36$, $19.85,22.60,31.65 \mathrm{~cm}$, respectively and in case of correlation analysis, a significant correlation was found between them. Similar significant result was found in case of BR28 (Table 1). Yield was significantly and positively associated with panicle length (Yadav et al. 2010). We also found that flag leaf length was positively associated with panicle length, thereby indicating associated with grain yield.

Variable amount of chlorophyll content was observed in the rice cultivars. The cultivar BR3 contained 50.54 and $37.61 \mathrm{mg} / \mathrm{g}$ chlorophyll for FL and PL, respectively. Similarly, BR4 contained 49.20 and 36.05 $\mathrm{mg} / \mathrm{g}$, BR11 41.12 and $32.17 \mathrm{mg} / \mathrm{g}$, BRRI dhan34 54.21 and $19.59 \mathrm{mg} / \mathrm{g}$ and BRRI dhan37 42.07 and 33.81 mg/g chlorophyll for FL and PL, respectively. Results indicating, in every variety FL always contained more chlorophyll than the PL. That means photosynthesis occurs mostly in FL and contributes mostly in panicle development. Prakash et al. (2011) found that the grain yield was positively related with flag leaf area in rice cultivars. Roy and Kar (1992), Gupta et al. (1999) also reported significant positive association between boot leaf length and grain yield per plant.

The weight of 100 grains was measured from the control and treated (flag leaf excised) plants. As in the Table 3, weight of 100 grains of BR3 was $2.75 \mathrm{~g}$ and $1.4 \mathrm{~g}$ for control and treated, respectively. Similarly, 100 grains weight of BR4 had $2.60 \mathrm{~g}$ and $1.7 \mathrm{~g}, \mathrm{BR} 11$ had $2.57 \mathrm{~g}$ and $1.8 \mathrm{~g}$, BRRI dhan34 had $3.1 \mathrm{~g}$ and 1.65 g, BRRI dhan37 had $2.31 \mathrm{~g}$ and $1.5 \mathrm{~g}$ for control and treated, respectively. Results indicating yield was always significantly reduced when flag leaf was removed from the plant at anthesis (Table 3). This is might be due to the inaproperiate food supply to sink that mostly flag leaf does and thus the effect was observed as various defects in the floral identity. Therefore, it is worth mentioning that flag leaf has a great contribution on yield.

We have also successfully counted the pollen, and differentiated viable pollens from non-viable one. When we have counted the percentage of viability of 91\% in BR3, 65\% in BR4, 82\% in BR11, 70\% in BRRI dhan34, and $80 \%$ in BRRI dhan 37 .

\section{Conclusion}

In rice, the flag leaf is metabolically active and critically important in determining yield. It has been assigned an important role in terms of supply of photosynthates to the grains. Any damage done to that leaf will have a direct and dramatic impact on crops potential. Therefore, it is important to protect the flag leaf in the early stages of crop. Raising the photosynthetic capacity of flag leaf is the key to 
overcome the photosynthate-source restriction on grain yield and to make a new breakthrough of yield potential in future development of rice.

Acknowledgements: The authors are gratefully acknowledged to UGC of the Government of the Peoples Republic of Bangladesh for their financial supports.

\section{References}

Abou-khalifa A, Misra AN and Salem KMA. 2008. Effect of leaf cutting on physiological traits and yield of two rice cultivars. African J Plant Sci. 2: 147-150.

Asana RD. 1968. In quest of yield. Indian J. Plant Physiol. 11: 1-10.

Ashraf M, Akbar M and Salim M. 1994. Genetic improvement in physiological traits of rice yield. In: Slafer G A, eds. Genetic Improvement of Field Crops. Marcel Dekker Incorporate, New York, Pp 413-455.

Ashrafuzzaman MM, Islam R, Ismail MR, Shahidullah SM and Hanafi MM. 2009. Evaluation of six aromatic rice varieties for yield and yield contributing characters. Int. J. Agric. Biol. 11: 616-620.

Bing Y, Wei-Ya X, Li-Jun L and Yong-Zhong X. 2006. QTL analysis for flag leaf characteristics and their relationships with yield and yield traits in rice. Acta Genetica Sinica 33: 824-832.

BRRI. 1999. Bangladesh Rice Research Institute, Modern Rice Cultivar 5: 11-18.

Chen WF, Xu ZJ, Zhang BL. 1995. Physiological bases of super high yield breeding in rice. Shenyang: Liaoning science and technology publishing company; Pp.1-2.

Chory J, Peto CA, Ashbaugh M, Saganich R, Pratt L and Ausubel F. 1989. Different roles for phytochrome in etiolated and green plants deduced from characterization of Arabidopsis thaliana mutants. Plant Cell 1: 867-880.

Choudhry AR, Shah AH, Ali L and Bashir M. 1986. Path coefficient analysis of yield and yield components in wheat. Pak. J. Agric. Res. 7: 71-75.

Davis MS, Forman A and Fajer J. 1979. Ligated chlorophyll cation radicals: their function in photosystem II of plant photosynthesis. Proc. Natl. Acad. Sci. USA 76: 4170-4174.

Food and Agriculture Organization of the United Nations (FAO). 1995. quarterly bulletin of statistics 8: 1-2.

Gupta A, Sharma RK, Mani VP and Chauhan VS. 1999. Variability and association analysis for grain yield and its components in hill races. J. Hill Res. 12: 99-101.

Khush GS and Toenniessen GH. 1991. Rice Biotechnology, International Rice Research Institute 6: vii-viii.

Li Z, Pinson SRM, Stansel JW and Paterson AH. 1998. Genetic dissection of the source-sink relationship affecting fecundity and yield in rice (Oryza sativa L.). Mol. Breed. 4: 419-426.
Lopez MT and Virmani SS. 2000. Development of TGMS lines for developing two- line rice hybrids for the tropics, Euphytica 144: 211-215.

Mackinney G. 1941. Absorption of light by chlorophyll solutions. J. Biol. Chem. 140: 315-322.

Misra AN.1987. Physiological aspects of grain formation in sorghum and pearl millet. In: Production technology for sorghum and pearl millet. ICAR/Sukhadia University, Jaipur, India, pp. 1-6.

Ohno Y .1976. Varietal differences of photosynthetic efficiency and dry matter production in indica rice. Tro. Agri. 53: 115-123.

Ortega BR, Aresti EM and Pereira RI. 2011. Implementation and evaluation of an image analysis system for determining viability of pollen grains in temperate rice. Chilean J. Agric. Res. 71: $16-22$.

Padmaja RS. 1991. Influence of source and sink on the production of high density grain and yield in rice. Indian J. Plant Physiol. 34: 339-348.

Prakash M, Anandan A and Kumar BS. 2011. Varietal variations in flag leaf area and yield in mutant lines of PY 5 rice. Karnataka J. Agric. Sci. 24: 525-526.

Raj A and Tripathi MP. 2000. Varietal variations in flag leaf area and yield in deep water rice. Indian J. Plant Physiol. 5: 293-295.

Roy A and Kar MK. 1992. Variability and correlation studies on upland rice. Oryza 29: 195-199.

Ramadas VS and Rajendrudu G. 1977. The photosynthetic efficiency of flag leaf in relation to structural features in some crop plants. Indian J. Plant Physiol. 22: 123-128.

Sheela G, Shai VN and Saran. 1990. Role of flag leaf on grain yield and spikelet sterility in rice cultivars. Oryza 27: 87-88.

Shen YG. 1980. Photosynthesis and matter production. China agriculture publishing company, Beijing, China. 31-32 pp.

Tambussi EA, Bort J, Guiamet JJ, Nogués S and Araus JL. 2007. The photosynthetic role of ears in C3 cereals: metabolism, water use efficiency and contribution to grain yield. Crit. Rev. Plant Sci. 26: 1-16.

Xu DQ and Shen YG. 1994. Progress on physiology of crop high production and high efficiency. Science publishing company, Beijing, China.17-23 pp.

Yadav SK, Suresh BG, Pandey P and Kumar B. 2010. Assessment of genetic variability, correlation and path association in rice (Oryza sativa L.). J. bio-sci. 18: 1-8.

Yoshida S. 1981. Fundamentals of rice crop science. International Rice Research Institute, Manila, Philippines. Los Baños, Laguna. 269 p.

Yue B, Xue WY, Luo LJ and Xing YZ. 2006. QTL analysis for flag leaf characteristics and their relationships with yield and yield traits in rice. Acta Genetica Scinica 33: 824-832.

Manuscript received on 7 November 2013 and revised on 8 December 2013 